\title{
The study of a new family of phase masks for three-dimensional fluorescence nanoscopy
}

\author{
Aleksei Gorshelev 1,*, Ivan Eremchev ${ }^{1,2}$, Sergei Kulik ${ }^{1}$, Andrei Naumov ${ }^{1,2,3}$, Evgeniy \\ Vorontsov $^{4}$, Vladimir Volostnikov ${ }^{4}$, and Svetlana Kotova ${ }^{4}$ \\ ${ }^{1}$ Institute of Spectroscopy RAS, 108840 Troitsk, Moscow, Russia \\ ${ }^{2}$ Moscow Institute of Physics and Technology, 141700 Dolgoprudny, Moscow region, Russia \\ ${ }^{3}$ Moscow State Pedagogical University, 119991 Moscow, Russia \\ ${ }^{4}$ Lebedev Physical Institute, 443011, Samara, Russia
}

\begin{abstract}
The question of the axial coordinate determination accuracy is quite sophisticated in far field 3D fluorescence nanoscopy. The accuracy of a point emitter axial coordinate reconstruction depends among other things on the conversion efficiency of the phase mask and the microscope objective instrumental function. It was found that the instrumental functions of different microscope objectives differ significantly from each other, most have a strongly non-uniform spatial distribution of the radiation intensity in a parallel beam created by an objective focused on a point emitter. It was shown that taking into account the actual microscope objective instrumental function when calculating the phase masks allows to increase significantly the axial coordinate reconstruction accuracy.
\end{abstract}

Fluorescence microscopy with ultra-high spatial resolution (far field fluorescence nanoscopy, FFN) is currently one of the most effective and in-demand tools for research in the fields of biological and medical physics, biology, and chemical physics. It is also of interest for applications in solid state physics. Many of these far field fluorescence nanoscopy techniques (STORM, PALM, etc.) are based on the possibility of fluorescence imaging of single point-like emitting labels with nanoscale reconstruction of their lateral spatial coordinates, and subsequent computer rendering of object nanostructure by mapping plenty single labels positions (see [1,2] and references therein). Application of the technique based on the theory of spiral beam optics allows to reconstruct all three spatial coordinates of a single point emitter with nanometer accuracy. In this technique the spatial phase modulation of the light beam is used for the transformation of the single emitter point spread function into double helix point spread function [3].

The accuracy of a point emitter axial coordinate reconstruction depends on a variety of experiment parameters: the number of recorded photons, the conversion efficiency of a phase mask, the microscope objective instrumental function, and the characteristics of the optical scheme used in the registration channel.

FFN methods can be especially informative if fluorescent single molecules (SM) of organic dyes are used as emitting point light sources at cryogenic temperatures, when zero-

\footnotetext{
* Corresponding author: gorshelev@isan.troitsk.ru
} 
phonon lines (ZPL) which correspond to purely electronic transitions in SMs are reachable for the observation. First, the analysis of ZPL of single molecules makes it possible to study the dynamic processes in impurity solid media, since the ZPL parameters are sensitive to the SM local environment characteristics. Second, the very large ratio of inhomogeneous absorption bandwidth to the very narrow ZPL homogeneous spectral width gives the opportunity to measure the fluorescence excitation spectra and coordinates for a huge number (up to all) efficiently fluorescing SMs in doped bulk solid samples - crystals, glasses, polymers (see $[1,2]$ and references therein).

To perform measurements at cryogenic temperatures, it is necessary to use special microobjectives designed for the operation at liquid-helium temperature, including in superfluid helium. In the case of an ideal microscope objective the propagation of light from a single point emitter located in a focal plane will be described after the objective by a plane wave with a Gaussian spatial intensity profile. However, in reality there are a variety of aberrations that degrade the picture. Manufacturers of the microscope objectives try to compensate for certain aberrations, depending on the tasks for which an objective is calculated. As a result, in most cases the spatial distribution of the radiation intensity in a parallel beam created by an objective focused on a point emitter, - the microscope objective instrumental function, is significantly different from the ideal one. In particular, it is peculiar to micro-objectives designed to operate at cryogenic temperatures.

In the case of such micro-objectives the use of phase masks calculated for the spatial distribution of the radiation intensity with a flat (or Gaussian) profile gives a low efficiency of conversion of the microscope objective instrumental function to the two-lobe function. In this connection the actual task is the calculation of new effective phase masks taking into account the shape of the microscope objective instrumental function.

In the present work with the use of single-crystal semiconductor colloidal quantum dots (QDs) as point emitters of radiation the instrumental functions of a number of microobjectives were measured and analysed. It was found that the instrumental functions of different micro-objectives differ significantly from each other, most have a strongly nonuniform spatial distribution of the radiation intensity in a parallel beam. With the use of an objective with the radiation intensity spatial distribution profile closest to a flat one, for a number of original calculated phase masks [4] the efficiency of the microscope objective instrumental function to the two-lobe function conversion was estimated. It was found that taking into account the actual microscope objective instrumental function when calculating the phase masks allows to increase significantly the conversion efficiency.

It was also shown that during the calculation of the optimal phase mask, it is necessary to take into account the actual diameter of the light beam and the pixel size of the phase modulator. Calibration curves - the correspondence between the two-lobe function rotation angle and the position of the radiating QD along the optical axis (axial coordinate) - were measured for a number of phase masks using specially developed technique. In addition, the question of the accuracy of the QD axial coordinate reconstruction was experimentally investigated.

This work was financially supported by RFBR (project 16-29-11809).

\section{References}

1. A. V. Naumov, Physics Uspekhi, 56, 605 (2013)

2. A. V. Naumov, I. Yu. Eremchev, A. A. Gorshelev, Europ. Phys. J. D, 68, 348 (2014)

3. S. R. P. Pavani, R. Piestun, Opt. Express, 16, 3484 (2008)

4. E. N. Vorontsov, S. P. Kotova, N. N. Losevsky, D. V. Prokopova, S. A. Samagin, Bull. Lebedev Phys. Inst., 45, 71 (2018) 